\title{
Socioeconomic Status in Pregnant Women and Sleep Quality During Pregnancy
}

\author{
Livier J. Silva-perez ${ }^{1}$, Natalia Gonzalez-Cardenas ${ }^{2}$, Sara Surani ${ }^{3}$, FA Etindele Sosso ${ }^{4,5}$, Salim R. Surani ${ }^{6,}$
} 7,8

1. Medicine, Autonomous University of Baja California, Tijuana, MEX 2. Medicine, Universidad Central de Venezuela, Caracas, VEN 3. Global Health, Harvard University, Cambridge, USA 4. Center of Advanced Research in Sleep Medicine, Hopital du Sacre-Coeur de Montreal, Montreal, CAN 5. Institut Santé Et Société, Université du Québec à Montréal, Montreal, CAN 6. Internal Medicine, Texas A\&M Health Science Center, Temple, USA 7. Internal Medicine, Corpus Christi Medical Center, Corpus Christi, USA 8. Internal Medicine, University of North Texas, Dallas, USA

Corresponding author: Salim R. Surani, srsurani@hotmail.com

\begin{abstract}
This review focuses on factors contributing to sleep quality among pregnant women with low socioeconomic statuses during the third trimester of their pregnancy. Electronic searches were conducted, following the Preferred Reporting Items for Systematic Reviews and Meta-Analysis (PRISMA) guidelines. We searched for published, peer reviewed, English language primary research articles using electronic databases including PubMed, EMBASE, Ovid, MEDLINE and Google Scholar ending June 2019. All references were reviewed manually and independently by authors. After applying the inclusion criteria, 56 articles were selected; 38 of which are full-text and included in this review. All articles related to the analysis of poor sleep quality among uncomplicated pregnant women were included. Pregnant women with a specific pathology were excluded.
\end{abstract}

We found poor sleep quality among pregnant women is correlated with low socioeconomic levels. Pregnant women with lower incomes tend to have inadequate diets, which further complicates the health of the mother and the baby. External factors including low income, poor quality of life and poor diet tend to increase the possibility of future health complications in both mother and child, and can result in complications such as preterm labor, low birth weight, preeclampsia, perinatal death, and spontaneous abortion.

Received 11/04/2019 Review began 11/04/2019 Review ended 11/13/2019 Published 11/18/2019

๑) Copyright 2019 Silva-perez et al. This is an open access article distributed under the terms of the Creative Commons Attribution License CC-BY 3.0., which permits unrestricted use, distribution, and reproduction in any medium, provided the original author and source are credited.
Categories: Family/General Practice, Quality Improvement, Public Health

Keywords: poor quality of sleep, pregnancy, low socioeconomic status, sleep in pregnancy

\section{Introduction And Background}

Sleep is a natural state of altered consciousness where the nervous system is relatively inactive. Sleep quality and duration are important for a number of physiological functions [1]. During pregnancy, combination of hormonal effects, mechanical effects due to enlarging uterus and the circulatory changes causes significant alteration in the sleep quality. Study by Mindell et al. in their survey of 2427 pregnant women showed that $76 \%$ of women reported poor sleep quality and 38\% had insufficient sleep [2]. According to National Sleep Foundation, in order to achieve good sleep quality, an individual must sleep at least $85 \%$ of the time in bed, should fall asleep within the first 30 minutes and should not wake up, no more than once during the night. If the individual wakes up, he or she should be able to fall back to sleep within 20 minutes $[3]$.

During pregnancy there is also alteration in the normal sleep with less slow wave sleep and increase sleep fragmentation [4]. In addition, alteration in sleep can have significant health and cardiovascular events. The association between pregnancy and sleep disturbances varies from insomnia, nighttime awakenings, parasomnias, and snoring. These sleep alterations are a consequence of hormonal and physical changes related to pregnancy. Moreover, poor sleep quality from physiological or pathological causes seems to be more prevalent during the third trimester of pregnancies $[5,6]$. During pregnancy, many physiological changes occur to achieve a homeostatic equilibrium for the benefit of the fetus. Particularly, the sleep adaptive modifications are present in the respiratory and cardiovascular systems, which are constantly responding to a myriad of hormonal events [7]. For example, increases in beta-human chorionic gonadotropin (HCG) and progesterone change sleep patterns. While estrogen shortens the duration of rapid eye movement (REM)-sleep, progesterone stimulates sleep by balancing the duration of the REM sleep stage and non-REM (NREM) stage for the health of the mother [8]. Additionally, estrogen influences an increase of secretions, vascularity, and edema in the respiratory tract. Moreover, a decrease of functional residual capacity and the dimension of airways results in shortness of breath. Rising levels of cortisol influence the activation of sympathetic tones, resulting in low vascular peripheral resistance and higher maternal heart rates. In summary, as time passes, these changes contribute to alterations of pre-pregnancy sleep patterns including shorter sleep durations, night awakenings, snoring, and gasping [9]. 
During pregnancy, alterations of sleep patterns are common. Prevalence varies widely from 30 to $78 \%$ across studies [10-12]. Beebe et al. characterized the symptoms experienced in late pregnancy, and reported that $68 \%$ of women had sleep disturbances and scored these disturbances highest for severity, frequency and distress [12]. Similarly, Hutchison et al. found that women reported both decrease in hours of sleep (8.1 to $7.5 \mathrm{~h}$ ) and poor quality of sleep (up to 61\%) during their third trimesters [9]. Likewise, a survey of 2427 women reported that pregnant women had poor quality of sleep (76\%), insomnia (57\%), night awakening (100\%) and sleep-breath alterations (19\%) from the beginning until the end of their pregnancies [2].

In a case-control study by Sut et al. from Turkey, sleep quality among pregnant women was poor than in their non-pregnant peers [13]. Socioeconomic status is a concept which considers the level of education, income, and occupation of the individual. There is a considerable evidence that low socioeconomic status is linked to obstetrical complications such as preterm deliveries, high rate of caesarean sections and third trimester hemorrhages [14].

Prior studies suggest that low-income pregnant women were at high risk of poor quality of sleep when compared with high-income pregnant women $[15,16]$. The spectrum of sleep disorders in developed countries is associated with poor maternal-fetal outcome. It seems that health care providers may overlook sleep disorders as a common complaint during gestation and shortly after delivery.

Understanding sleep patterns during pregnancy may have significant impacts on gestational outcomes [17]. During pregnancy, poor quality of sleep (PQOS) and sleep disorders have been associated with hypertensive states like preeclampsia (Preeclampsia can occur as early as 20 weeks of gestation, if one is at risk than symptoms usually occurs around 34th week) [18,19]. Short sleep duration has also been linked to the development of gestational diabetes, high risk of preterm labor, and cesarean delivery [20,21]. Furthermore, Dolatian et al. reports that lower socioeconomic status and high levels of stress have shown correlation with lower birth-weights [22].

Despite data suggesting sleep disturbances among pregnant patients, the influence of socioeconomic status during last months of pregnancy is not clearly understood [21, 23-25]. This mini review focuses on presenting factors contributing to poor sleep quality among pregnant patients with lower income and socioeconomic statuses, especially during their third trimesters.

\section{Review}

\section{Methods}

We conducted an electronic-based search following the Preferred Reporting Items for Systematic Reviews and Meta-Analysis (PRISMA) guidelines. We searched for published, peer reviewed, English language primary research articles using electronic databases as PubMed, EMBASE, Ovid, MEDLINE, and Google Scholar. The following medical subject heading (Mesh) terms, keywords, and their combinations were used: "pregnancy", "low socioeconomic status", "poor quality of sleep”. The search was limited to studies published up until June 2019. We manually searched the reference lists of identified studies. We included all original articles as well as systematic reviews. Three authors (LS, NG, SS) assessed each article independently. Our search, after excluding duplicates, non-English articles, non-related citations to key words, and unavailable full text was filtered to 38 articles. These articles included randomized studies, cross sectional observational studies, quasi experimental studies and case control studies. One hundred articles in total were reviewed (See Figure 1). 


\section{Cureus}

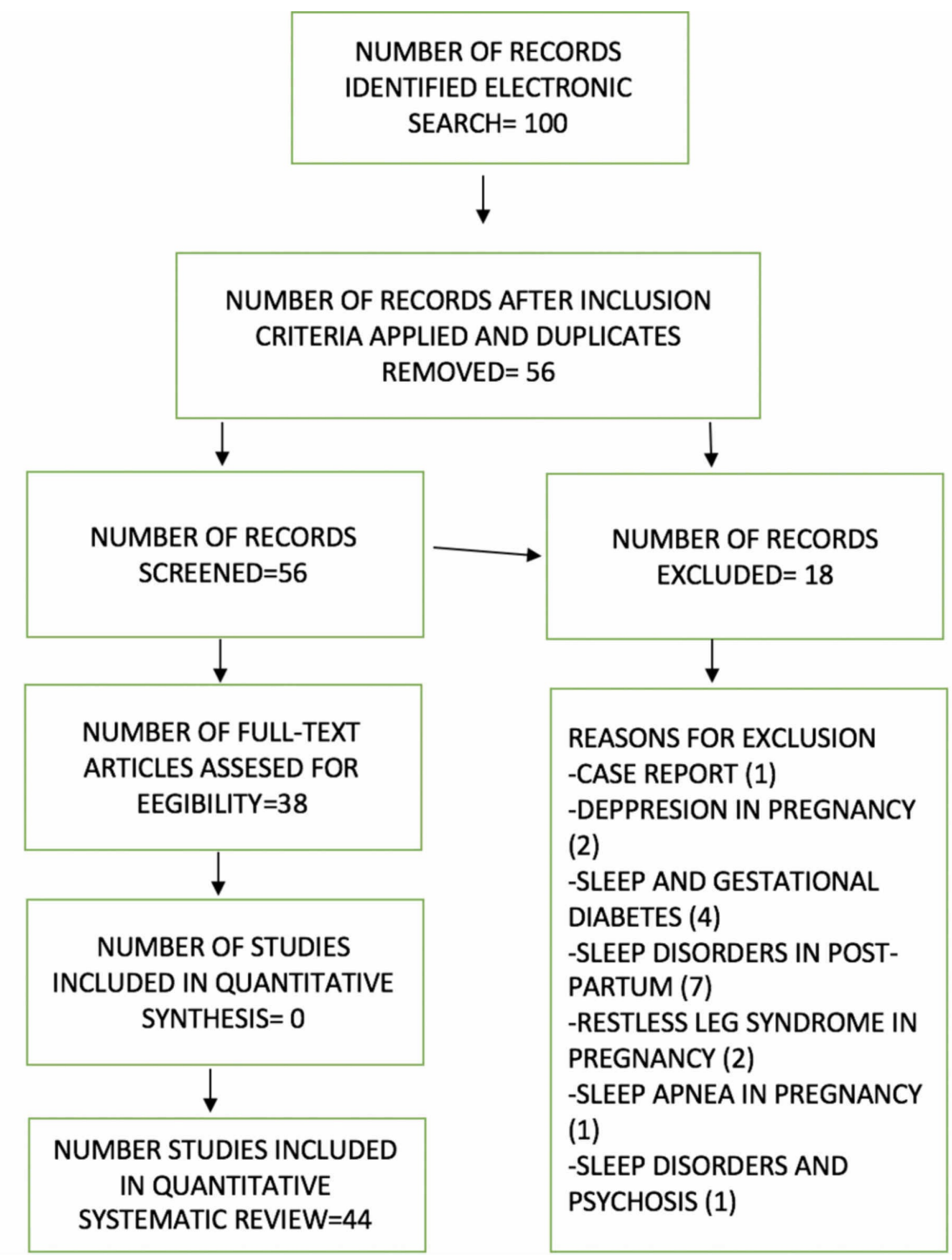

FIGURE 1: PRISMA flow chart.

PRISMA: Preferred Reporting Items for Systematic Reviews and Meta-Analysis

\section{Discussion}

The sleep time required for a pregnant woman is approximately 7.5 hours or more [26]. The Pittsburg Classification Sleep Quality Index was the most commonly used instrument to measure sleep quality [27]. These questions focus on assessing the quality of sleep based on duration, disturbances, latency, daytime dysfunction, efficiency, and quality among other characteristics.

There are several factors that affect sleep quality during pregnancy; socioeconomic levels being the only indicator that also affects the quality of life of the pregnant woman. Additionally, when sleep is interrupted by physical-hormonal-psychological changes, one can see poor outcomes in both the woman and the newborn [16].

According to the American Community Survey 2013-2017, the average household income in the United States was $\$ 57,652$ [28]. A household income less than $\$ 50,000$ per year is considered a low socioeconomic status. It is well known that Medicaid is an important health care service for pregnant women with lower incomes and that Medicaid is responsible for health coverage of approximately $50 \%$ of all births in the United States [2]. Moreover, a recent study reported that premature births and poor health are often observed in women who receive Medicaid [29]. To further complicate matters, pregnant women with low income have a higher probability of presenting long-term conditions during the gestation process [30].

Sleeping through the night during pregnancy is crucial for the health of both the mother and the fetus. At the same time, it is challenging to get sufficient sleep because of interruption by socioeconomic, physical, and 


\section{Cureus}

psychological factors. According to the Sedov et al., in the third trimester of gestation, insomnia is present more often due to muscle-skeletal pain, overweight physique, restless legs, reflux, non-comfortable positions and snoring [31]. Night awakenings during the third trimester are more frequent due to an uncomfortable muscle-skeletal pain [32]. In a cross-sectional study in pregnant women, back pain was present in 55\% to $75 \%$ of pregnant women during pregnancy. Mirroring these observations, Yikar and Nazik also found that the most common complaints during pregnancy are seen in second and third trimester. Furthermore, the symptoms reported during the second and third trimester were fatigue, exhaustion, restless legs, backache, nocturia, depression, and anxiety episodes [33].

Dolatian et al. found that women who sleep less than 8 hours have a higher risk of cesareansection deliveries, preterm labor, and decrease in baby size when compared with their gestational age increases by 2.2 fold [34]. Another study showed that pregnant women with Medicaid have a higher probability of C-section or preterm deliveries [35]. Moreover, Bruce also showed that pregnant women with low socioeconomic status or financial insecurity demonstrate a tendency to have a poor quality of life which affects the health of pregnant women [36]. Table 1 illustrates the findings of some of the key studies suggesting sleep disturbances during pregnancy and also the effects of socioeconomic status. 


\section{Cureus}

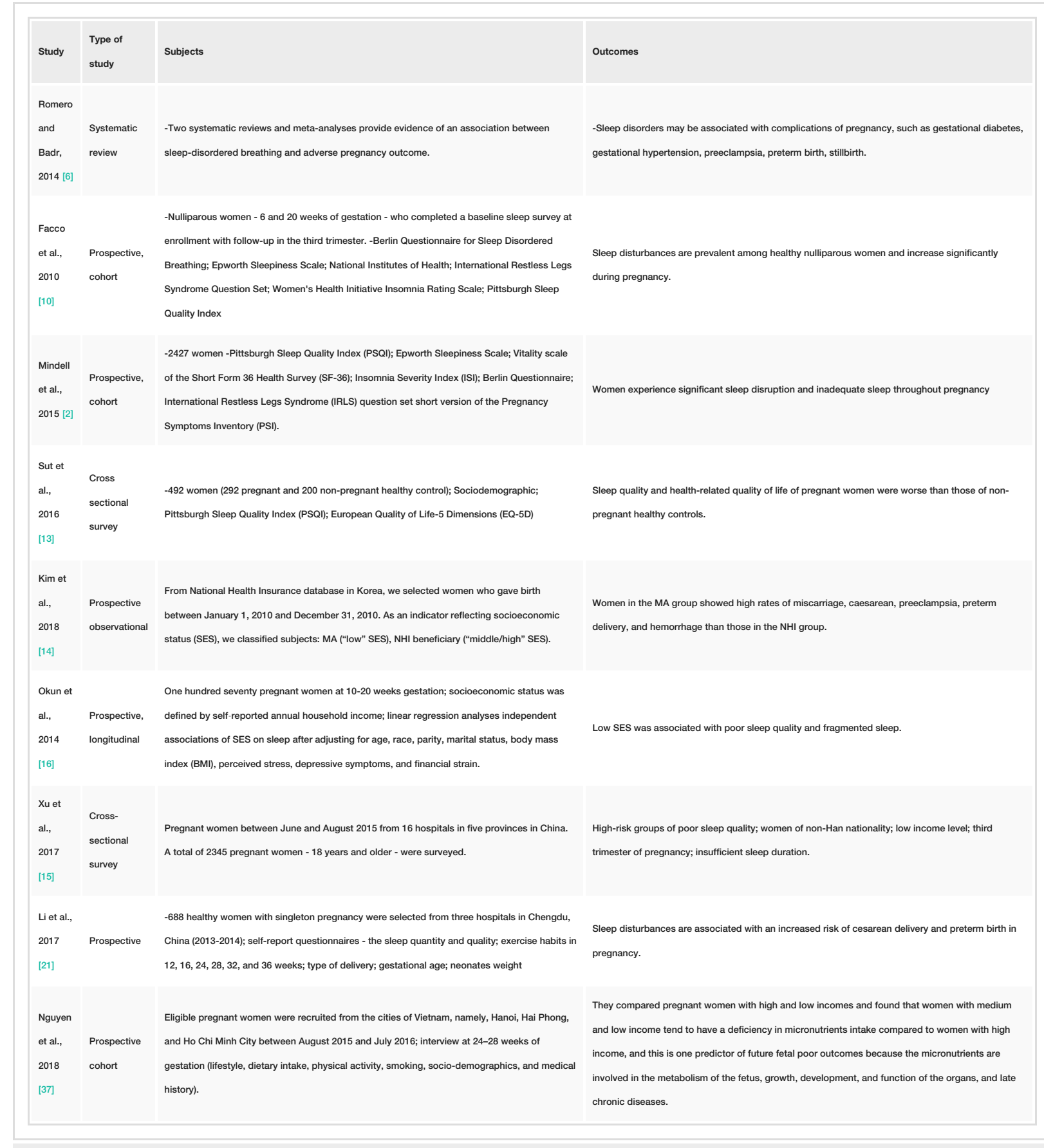

TABLE 1: Studies suggesting sleep disorder during pregnancy.

Pregnant women with low socio-economic status tend to receive inadequate nutrients [37]. Another article found how women with lower incomes have poorer diets and consume higher levels of saturated fatty, carbohydrates, soft beverages, or food without adequate nutrients. An investigation shows how high fatty food consumption, especially in women with overweight physiques, increases the possibility to develop obesity, gestational diabetes, abortion, preeclampsia. There is also a high possibility that the baby will develop diabetes mellitus type 2 in their adulthood [38]. The European Food Safety Authority 2014 recommends that pregnant women have an additional calorie intake in every trimester. For example, the 1st trimester should be $70 \mathrm{kcal}$ per day, 2nd trimester should be 260 to $500 \mathrm{kcal}$ per day, and 3rd trimester should be $500 \mathrm{kcal}$ per day, to maintain a balance due to increase in demands during pregnancy [39].

According to the World Health Organization, in order to improve maternal and fetal wellbeing, a balanced intake during gestation must contain the following nutrients: green leafy vegetables, protein (fish, salmon, meat), cereal, beans, and nuts [40]. The salmon contains an elevated quantity of Omega 3 fatty acid, which plays an important role in the brain and retina formation of the fetus and also reduces the possibility of preterm delivery [41]. Iodine is a micronutrient required for metabolic and hormonal functions during 
pregnancy. Decreased intake may cause abortion, brain damage in the fetus, or mortality in the perinatal period [42]. Calcium is involved in fetus development, important for normal birth weight, decreases the risk of premature delivery, and controls blood pressure during pregnancy [43]. Folic acid consumption is necessary for preventing neuro-tube congenital defects or heart disease, and also helps with adequate placenta formation [44].

RDA Italy recommends regular intake of these micronutrients for positive benefits during the gestational process: Omega 3 (250 mg), Iodine (250 mg), Calcium (1200 mg), Folic acid (600 mg), Vitamin D (15 mg). All these nutrients optimize the requirements during pregnancy [39].

\section{Conclusions}

In this review, we focused on how low socioeconomic status affects the quality of sleep in pregnant women during and after the third trimester of pregnancy. As in our discussion, we suggested that factors involved in the poor quality of sleep during pregnancy are related, and how low socioeconomic levels are associated with poor quality of life. The controlled trials of high quality though are lacking in these areas. A pregnant woman with a low income who tends to have an inadequate diet may have future health complications for both herself and her fetus. During pregnancy, the body undergoes physical, hormonal, and physiological changes that are magnified by external factors including low income, poor quality of life, and poor diet. These factors tend to increase the possibility of future health outcomes in both, mother and fetus and can result in preterm labor, low birth weight, preeclampsia, perinatal death, and spontaneous abortion. Further research is needed to evaluate the differences between high- and low-income groups in comparison to their non-pregnant peers. Clinicians should take advantage of assessing instruments of quality of sleep to identify pregnant women at risk of poor perinatal outcomes. Nutritionists should asses and identify pregnant women with high risk for future poor outcomes.

\section{Additional Information \\ Disclosures}

Conflicts of interest: In compliance with the ICMJE uniform disclosure form, all authors declare the following: Payment/services info: All authors have declared that no financial support was received from any organization for the submitted work. Financial relationships: All authors have declared that they have no financial relationships at present or within the previous three years with any organizations that might have an interest in the submitted work. Other relationships: All authors have declared that there are no other relationships or activities that could appear to have influenced the submitted work.

\section{References}

1. WHO technical meeting on sleep and health. (2004). Accessed: August 03, 2019: http://www.euro.who.int/_data/assets/pdf_file/0008/114101/E84683.pdf.

2. Mindell JA, Cook RA, Nikolovski J: Sleep patterns and sleep disturbances across pregnancy. Sleep Med. 2015, 16:483-488. 10.1016/j.sleep.2014.12.006

3. What is a good quality sleep? . (2019). Accessed: October 28, 2019: https://www.sleepfoundation.org/pressrelease/what-good-quality-sleep.

4. Oyiengo D, Louis M, Hott B, Bourjeily G: Sleep disorders in pregnancy. Clin Chest Med. 2014, 35:571-587. 10.1016/j.ccm.2014.06.012

5. Christian LM, Carroll JE, Porter K, Hall MH: Sleep quality across pregnancy and postpartum: effects of parity and race. Sleep Health. 2019, 5:327-334. 10.1016/j.sleh.2019.03.005

6. Romero R, Badr MS: A role for sleep disorders in pregnancy complications: challenges and opportunities . Am J Obstet Gynecol. 2014, 210:3-11. 10.1016/j.ajog.2013.11.020

7. Pien GW, Schwab RJ: Sleep disorders during pregnancy. Sleep. 2004, 27:1405-1417. 10.1093/sleep/27.7.1405

8. Robertson NT, Turner JM, Kumar S: Pathophysiological changes associated with sleep disordered breathing and supine sleep position in pregnancy. Sleep Med Rev. 2019, 46:1-8. 10.1016/j.smrv.2019.04.006

9. Hutchison BL, Stone PR, McCowan LM, Stewart AW, Thompson JM, Mitchell EA: A postal survey of maternal sleep in late pregnancy. BMC Pregnancy Childbirth. 2012, 12:144. 10.1186/1471-2393-12-144

10. Facco FL, Kramer J, Ho KH, Zee PC, Grobman WA: Sleep disturbances in pregnancy. Obstet Gynecol. 2010, 115:77-83. 10.1097/AOG.0b013e3181c4f8ec

11. Sarberg M, Bladh M, Josefsson A, Svanborg E: Sleepiness and sleep-disordered breathing during pregnancy. Sleep Breath. 2016, 20:1231-1237. 10.1007/s11325-016-1345-9

12. Beebe KR, Gay CL, Richoux SE, Lee KA: Symptom experience in late pregnancy. J Obstet Gynecol Neonatal Nurs. 2017, 46:508-520. 10.1016/j.jogn.2017.03.007

13. Sut HK, Asci O, Topac N: Sleep quality and health-related quality of life in pregnancy . J Perinat Neonatal Nurs. 2016, 30:302-309. 10.1097/JPN.0000000000000181

14. Kim MK, Lee SM, Bae SH, et al.: Socioeconomic status can affect pregnancy outcomes and complications, even with a universal healthcare system. Int J Equity Health. 2018, 17:2. 10.1186/s12939-017-0715-7

15. Xu X, Liu D, Zhang Z, Sharma M, Zhao Y: Sleep duration and quality in pregnant women: a cross-sectional survey in China. Int J Environ Res Public Health. 2017, 14:817. 10.3390/ijerph14070817

16. Okun ML, Tolge M, Hall M: Low socioeconomic status negatively affects sleep in pregnant women . J Obstet Gynecol Neonatal Nurs. 2014, 43:160-167. 10.1111/1552-6909.12295

17. Hux VJ, Roberts JM, Okun ML: Allostatic load in early pregnancy is associated with poor sleep quality . Sleep Med. 2017, 33:85-90. 10.1016/j.sleep.2016.09.001 
18. Wilson DL, Walker SP, Fung AM, Pell G, O'Donoghue FJ, Barnes M, Howard ME: Sleep-disordered breathing in hypertensive disorders of pregnancy: a BMI-matched study. J Sleep Res. 2018, 27:12656. 10.1111/jsr.12656

19. Dominguez JE, Habib AS, Krystal AD: A review of the associations between obstructive sleep apnea and hypertensive disorders of pregnancy and possible mechanisms of disease. Sleep Med Rev. 2018, 42:37-46. 10.1016/j.smrv.2018.05.004

20. Cai S, Tan S, Gluckman PD, et al.: Sleep quality and nocturnal sleep duration in pregnancy and risk of gestational diabetes mellitus. Sleep. 2017, 40:zsw058. 10.1093/sleep/zsw058

21. Li R, Zhang J, Zhou R, et al.: Sleep disturbances during pregnancy are associated with cesarean delivery and preterm birth. J Matern Fetal Neonatal Med. 2017, 30:733-738. 10.1080/14767058.2016.1183637

22. Dolatian M, Mahmoodi Z, Alavi-Majd H, Moafi F, Ghorbani M, Mirabzadeh A: Psychosocial factors in pregnancy and birthweight: path analysis. J Obstet Gynaecol Res. 2016, 42:822-830. 10.1111/jog.12991

23. Gonzalez R, Valenza MC, Molina CM, Torres Sanchez I, Cabrera Martos I, Gonzalez-Jimenez E: Sleep disturbances and gestational diabetes prevalence on last trimester of pregnancy. (Article in Spanish). Nutr Hosp. 2015, 32:1139-1144. 10.3305/nh.2015.32.3.9365

24. Dunietz GL, Lisabeth LD, Shedden K, et al.: Restless legs syndrome and sleep-wake disturbances in pregnancy. J Clin Sleep Med. 2017, 13:863-870. 10.5664/jcsm.6654

25. Guinhouya BC, Bisson M, Dubois L, Series F, Kimoff JR, Fraser WD, Marc I: Body weight status and sleep disturbances during pregnancy: does adherence to gestational weight gain guidelines matter?. J Womens Health. 2019, 28:535-543. 10.1089/jwh.2017.6892

26. Ozkan SA, Rathfisch G: The effect of relaxation exercises on sleep quality in pregnant women in the third trimester: a randomized controlled trial. Complement Ther Clin Pract. 2018, 32:79-84.

10.1016/j.ctcp.2018.05.008

27. Qiu C, Gelaye B, Zhong QY, Enquobahrie DA, Frederick IO, Williams MA: Construct validity and factor structure of the Pittsburgh Sleep Quality Index among pregnant women in a Pacific-Northwest cohort. Sleep Breath. 2016, 20:293-301. 10.1007/s11325-016-1313-4

28. United States Census Bureau: American Community Survey, 2013-2017. 5-Year Estimates . United States Census Bureau. 2017, Accessed: November 17, 2019: https://www.census.gov/newsroom/presskits/2018/acs-5year.html.

29. Center for Medicaid and CHIP Services. (2013). Accessed: November 17, 2019: https://www.medicaid.gov/Federal-Policy-Guidance/Downloads/SMD-13-002.pdf.

30. Singh GK, Daus GP, Allender M, et al.: Social determinants of health in the United States: addressing major health inequality trends for the nation, 1935-2016. Int J MCH AIDS. 2017, 6:139-164. 10.21106/ijma.236

31. Sedov ID, Cameron EE, Madigan S, Tomfohr-Madsen LM: Sleep quality during pregnancy: a meta-analysis. Sleep Med Rev. 2018, 38:168-176. 10.1016/j.smrv.2017.06.005

32. Mota MJ, Cardoso M, Carvalho A, Marques A, Sa-Couto P, Demain S: Women's experiences of low back pain during pregnancy. J Back Musculoskelet Rehabil. 2015, 28:351-357. 10.3233/bmr-140527

33. Yikar SK, Nazik E: Effects of prenatal education on complaints during pregnancy and on quality of life . Patient Educ Couns. 2019, 102:119-125. 10.1016/j.pec.2018.08.023

34. Dolatian M, Mehraban Z, Sadeghniat K: The effect of impaired sleep on preterm labour . West Indian Med J. 2014, 63:62-67. 10.7727/wimj.2012.305

35. Access in brief: pregnant women and Medicaid . (2019). Accessed: November 17, 2019: https://www.macpac.gov/publication/access-in-brief-pregnant-women-and-medicaid/.

36. Calikoglu EO, Akcay HB, Kavuncuoglu D, Tanriverdi EC, Yerli EB, Salcan S: Socio-demographic characteristics and quality of life among women in the 3rd trimester of pregnancy living in Erzurum city center. J Family Practice Palliat Care. 2018, 3:33-38. 10.22391/fppc.373910

37. Nguyen CL, Hoang DV, Nguyen PTH, et al.: Low dietary intakes of essential nutrients during pregnancy in Vietnam. Nutrients. 2018, 10:E1025. 10.3390/nu10081025

38. Bruce KD: Maternal and in utero determinants of type 2 diabetes risk in the young . Curr Diab Rep. 2014, 14:446. 10.1007/s11892-013-0446-0

39. Sette S, Le Donne C, Piccinelli R, Arcella D, Turrini A, Leclercq C: The third Italian National Food Consumption Survey, INRAN-SCAI 2005-06--part 1: nutrient intakes in Italy. Nutr Metab Cardiovasc Dis. 2011, 21:922-932. 10.1016/j.numecd.2010.03.001

40. Marangoni F, Cetin I, Verduci E, et al.: Maternal diet and nutrient requirements in pregnancy and breastfeeding. An Italian consensus document. Nutrients. 2016, 8:629. 10.3390/nu8100629

41. Lauritzen L, Carlson SE: Maternal fatty acid status during pregnancy and lactation and relation to newborn and infant status. Matern Child Nutr. 2011, 7:41-58. 10.1111/j.1740-8709.2011.00303.x

42. Trumpff C, Vandevijvere S, Moreno-Reyes R, Vanderpas J, Tafforeau J, Van Oyen H, De Schepper J: Neonatal thyroid-stimulating hormone level is influenced by neonatal, maternal, and pregnancy factors. Nutr Res. 2015, 35:975-981. 10.1016/j.nutres.2015.09.002

43. Hofmeyr GJ, Lawrie TA, Atallah AN, Duley L, Torloni MR: Calcium supplementation during pregnancy for preventing hypertensive disorders and related problems. Cochrane Database Syst Rev. 2014, 001059. 10.1002/14651858.CD001059.pub4

44. Cawley S, Mullaney L, McKeating A, Farren M, McCartney D, Turner MJ: A review of European guidelines on periconceptional folic acid supplementation. Eur J Clin Nutr. 2016, 70:143-154. 10.1038/ejcn.2015.131 